\title{
Extension of Meshless Galerkin/Petrov-Galerkin Approach without Using Lagrange Multipliers*)
}

\author{
Atsushi KAMITANI, Teruou TAKAYAMA, Taku ITOH $^{1)}$ and Hiroaki NAKAMURA ${ }^{2)}$ \\ Yamagata University, Yonezawa 992-8510, Japan \\ ${ }^{1)}$ Seikei University, Musashino 180-8633, Japan \\ ${ }^{2)}$ National Institute for Fusion Science, Toki 509-5292, Japan
}

(Received 5 December 2010 / Accepted 19 April 2011)

\begin{abstract}
By directly discretizing the weak form used in the finite element method, meshless methods have been derived. Neither the Lagrange multiplier method nor the penalty method is employed in the derivation of the methods. The resulting methods are divided into two groups, depending on whether the discretization is based on the Galerkin or the Petrov-Galerkin approach. Each group is further subdivided into two groups, according to the method for imposing the essential boundary condition. Hence, four types of the meshless methods have been formulated. The accuracy of these methods is illustrated for two-dimensional Poisson problems.
\end{abstract}

(C) 2011 The Japan Society of Plasma Science and Nuclear Fusion Research

Keywords: boundary-value problem, collocation, essential boundary condition, Lagrange multiplier, meshless method, weak form

DOI: $10.1585 /$ pfr.6.2401074

\section{Introduction}

The finite element method (FEM) has been applied to various fields in fusion engineering and plasma physics and, consequently, it has produced a number of fruitful results. Before using a numerical code based on the FEM, a region must be divided into a set of finite elements. The elements are introduced not only for the approximation of the region shape but also for the definition of the interpolants. In other words, the method for representing the geometric shape is closely related to the basis functions in the FEM. However, it is this relation that complicates the input data for a FEM code.

In order to resolve these difficulties of the FEM, many types of meshless methods have been so far proposed [1-6]. Among them, the Element-Free Galerkin (EFG) method [2] and the Meshless Local Petrov-Galerkin (MLPG) method [3] are most widely applied to numerical simulations. The major differences between both methods are listed as follows: the implementation method of essential boundary condition and selections of the function spaces containing test/trial functions. As the implementation method, the Lagrange multiplier method and the penalty method are adopted in the EFG and the MLPG, respectively.

Our earlier work [6] shows that, if the essential boundary condition is incorporated to the weak form by using the penalty method, the resulting algebraic equations become ill-conditioned. On the other hand, the Lagrange multi-

author'se-mail: kamitani@yz.yamagata-u.ac.jp

*) This article is based on the presentation at the 20th International Toki Conference (ITC20). plier cannot be compatible with the Petrov-Galerkin approach. Thus, for the purpose of unifying the EFG and the MLPG, neither the penalty method nor the Lagrange multiplier method can be used for implementing the essential boundary condition.

The purpose of the present study is to formulate the meshless Galerkin/Petrov-Galerkin approach without using the penalty method or the Lagrange multiplier method. To this end, the weak form and the essential boundary condition are separately discretized and, subsequently, the matrix equation is derived by means of the concept of an orthogonal complement.

\section{FEM-Type Weak Form}

For simplicity, we consider the following twodimensional (2D) Poisson problem on the domain $\Omega$ bounded by a simple closed curve $\partial \Omega$ :

$$
\begin{aligned}
-\nabla^{2} u & =p & & \text { in } \Omega, \\
u & =\bar{u} & & \text { on } \Gamma_{\mathrm{D}}, \\
\frac{\partial u}{\partial n} & =\bar{q} & & \text { on } \Gamma_{\mathrm{N}},
\end{aligned}
$$

where $\Gamma_{\mathrm{D}}$ and $\Gamma_{\mathrm{N}}$ are parts of $\partial \Omega$ such that $\Gamma_{\mathrm{D}} \cup \Gamma_{\mathrm{N}}=\partial \Omega$ and $\Gamma_{\mathrm{D}} \cap \Gamma_{\mathrm{N}}=\phi$. In addition, $\boldsymbol{n}$ denotes an outward unit normal on $\partial \Omega$. Furthermore, the superposed bar indicates prescribed boundary values and $p(\boldsymbol{x})$ is a given function on $\Omega$. Although the 2D Poisson problem is used to demonstrate the formulation of meshless methods, the discretization procedure used in the present study is applicable to general boundary-value problems.

After a straightforward calculation, we can prove that 
the above Poisson problem is equivalent to the variational problem of the functional,

$$
\begin{aligned}
I[u, \lambda] \equiv & \iint_{\Omega}\left(\frac{1}{2}|\nabla u|^{2}-p u\right) \mathrm{d}^{2} x-\int_{\Gamma_{\mathrm{N}}} \bar{q} u \mathrm{~d} s \\
& +\int_{\Gamma_{\mathrm{D}}} \lambda(u-\bar{u}) \mathrm{d} s .
\end{aligned}
$$

Here, $\lambda(s)$ is the Lagrange multiplier that is a function of an arclength $s$ along $\Gamma_{\mathrm{D}}$. From the stationarity condition of the functional $I[u, \lambda]$ with respect to $u$ and $\lambda$, we get the following weak form:

$$
\begin{aligned}
& { }^{\forall} \delta u^{\forall} \delta \lambda: J[\delta u, u] \\
& \quad+\int_{\Gamma_{\mathrm{D}}} \delta \lambda(u-\bar{u}) \mathrm{d} s+\int_{\Gamma_{\mathrm{D}}} \delta u \lambda \mathrm{d} s=0,
\end{aligned}
$$

where $J[w, u]$ is the functional defined by

$$
\begin{aligned}
J[w, u] \equiv & \iint_{\Omega} \nabla w \cdot \nabla u \mathrm{~d}^{2} \boldsymbol{x}-\iint_{\Omega} w p \mathrm{~d}^{2} \boldsymbol{x} \\
& -\int_{\Gamma_{\mathrm{N}}} w \bar{q} \mathrm{~d} s .
\end{aligned}
$$

Since $\delta u$ and $\delta \lambda$ are variations of the functions $u$ and $\lambda$, respectively, both $u$ and $\delta u$ must be contained in the same function space and, similarly, both $\lambda$ and $\delta \lambda$ must be also contained in the same function space. In this sense, not the Petrov-Galerkin but the Galerkin approach can be applied to the discretization of (5). In fact, Belytscheko [2] formulated the EFG method by discretizing (5) with the Galerkin approach. However, in order to solve the Poisson problem by means of the Petrov-Galerkin approach, we have to start a considerably different weak form.

As is well known, both (1) and (3) are satisfied if and only if the following weak form is fulfilled:

$$
{ }^{\forall} w \text { s.t. }\left.w\right|_{\Gamma_{\mathrm{D}}}=0: J[w, u]=0 .
$$

Here, ${ }^{\forall} w$ s.t. $\left.w\right|_{\Gamma_{\mathrm{D}}}=0$ denotes an arbitrary function $w(\boldsymbol{x})$ that satisfies $w=0$ on $\Gamma_{\mathrm{D}}$. Note that (6) includes not the essential boundary condition but the natural one. In the following, (6) is referred as to the FEM-type weak form. In the next section, we derive the meshless methods by applying the Galerkin/Petrov-Galerkin approach to (6).

\section{Meshless Methods}

In this section, the FEM-type weak form (6) is discretized with both the weight functions and the shape functions of the moving least-squares (MLS) approximation [1-3]. To this end, the nodes, $\boldsymbol{x}_{1}, \boldsymbol{x}_{2}, \cdots, \boldsymbol{x}_{N}$, are placed in $\Omega \cup \partial \Omega$ and, subsequently, the weight function $w_{i}(\boldsymbol{x})$ with a compact support is assigned to the $i$ th node $\boldsymbol{x}_{i}$. By using the weight functions, the shape functions $\phi_{i}(\boldsymbol{x})$ ( $i=1,2, \cdots, N)$ can be determined. In the following, the number of nodes on $\Gamma_{\mathrm{D}}$ is denoted by $K$. In addition, $\left\{\boldsymbol{e}_{1}^{*}, \boldsymbol{e}_{2}^{*}, \cdots, \boldsymbol{e}_{N}^{*}\right\}$ and $\left\{\boldsymbol{e}_{1}, \boldsymbol{e}_{2}, \cdots, \boldsymbol{e}_{K}\right\}$ are the orthonormal system of the $N$-dimensional vector space and that of the $K$-dimensional vector space, respectively. Also, let $\left\{\psi_{1}(\boldsymbol{x}), \psi_{2}(\boldsymbol{x}), \cdots, \psi_{N}(\boldsymbol{x})\right\}$ be a set of linearly independent functions on $\Omega$.

\subsection{Discretization}

In order to discretize (6), the test function $w(\boldsymbol{x})$ and the trial function $u(\boldsymbol{x})$ are assumed as

$$
w(\boldsymbol{x})=\sum_{i=1}^{N} \hat{w}_{i} \psi_{i}(\boldsymbol{x}), \quad u(\boldsymbol{x})=\sum_{i=1}^{N} \hat{u}_{i} \phi_{i}(\boldsymbol{x}) .
$$

After substituting (7) into $J[w, u]=0$, we get

$$
(\hat{\boldsymbol{w}}, A \hat{\boldsymbol{u}}-\boldsymbol{f})=0
$$

where $\hat{\boldsymbol{w}}$ and $\hat{\boldsymbol{u}}$ are defined by

$$
\hat{\boldsymbol{w}}=\sum_{i=1}^{N} \hat{w}_{i} \boldsymbol{e}_{i}^{*}, \quad \hat{\boldsymbol{u}}=\sum_{i=1}^{N} \hat{u}_{i} \boldsymbol{e}_{i}^{*} .
$$

In addition, $A$ and $\boldsymbol{f}$ are given by

$$
\begin{aligned}
& A=\sum_{i=1}^{N} \sum_{j=1}^{N} \iint_{\Omega} \nabla \psi_{i} \cdot \nabla \phi_{j} \mathrm{~d}^{2} \boldsymbol{x} \boldsymbol{e}_{i}^{*} \boldsymbol{e}_{j}^{* T}, \\
& \boldsymbol{f}=\sum_{i=1}^{N}\left(\iint_{\Omega} \psi_{i} p \mathrm{~d}^{2} \boldsymbol{x}+\int_{\Gamma_{\mathrm{N}}} \psi_{i} \bar{q} \mathrm{~d} s\right) \boldsymbol{e}_{i}^{*} .
\end{aligned}
$$

As is apparent from (6), the constraint $\left.w\right|_{\Gamma_{\mathrm{D}}}=0$ is imposed on the test function $w(\boldsymbol{x})$. Next, let us derive the discretized form of the constraint. To this end, the constraint is rewritten as the equivalent proposition:

$$
{ }^{\forall} \beta(s): \int_{\Gamma_{\mathrm{D}}} \beta(s) w(\boldsymbol{x}(s)) \mathrm{d} s=0 .
$$

Let $\left\{N_{1}(s), N_{2}(s), \cdots, N_{K}(s)\right\}$ be a set of linearly independent functions on $\Gamma_{\mathrm{D}}$. Then, the above proposition can be discretized as

$$
\left(\hat{\boldsymbol{w}}, \boldsymbol{c}_{p}\right)=0 \quad(p=1,2, \cdots, K),
$$

where $N$-dimensional vectors $\boldsymbol{c}_{p}$ 's are defined by

$$
\boldsymbol{c}_{p} \equiv \sum_{i=1}^{N} \int_{\Gamma_{\mathrm{D}}} \psi_{i}(\boldsymbol{x}(s)) N_{p}(s) \mathrm{d} s \boldsymbol{e}_{i}^{*} .
$$

Apparently, (10) indicates that $\hat{\boldsymbol{w}}$ is contained in the orthogonal complement of $V=\operatorname{span}\left(\boldsymbol{c}_{1}, \boldsymbol{c}_{2}, \cdots, \boldsymbol{c}_{K}\right)$. Hence, the FEM-type weak form (6) can be discretized as

$$
{ }^{\forall} \hat{\boldsymbol{w}} \in V^{\perp}:(\hat{\boldsymbol{w}}, A \hat{\boldsymbol{u}}-\boldsymbol{f})=0 .
$$

The above equation means that $A \hat{\boldsymbol{u}}-\boldsymbol{f} \in\left(V^{\perp}\right)^{\perp}=V$. In other words, there exists a $K$-dimensional vector $\hat{\boldsymbol{v}}$ such that

$$
A \hat{u}+C \hat{v}=f
$$

where $C \equiv\left[c_{1} c_{2} \cdots c_{K}\right]$.

Finally, the essential boundary condition (2) is discretized. By using the similar method used for the constraint $\left.w\right|_{\Gamma_{\mathrm{D}}}=0$, we obtain

$$
D^{T} \hat{\boldsymbol{u}}=\boldsymbol{g}
$$

where $D$ and $g$ are given by 


$$
\begin{aligned}
& D=\sum_{i=1}^{N} \sum_{p=1}^{K} \int_{\Gamma_{\mathrm{D}}} \phi_{i}(\boldsymbol{x}(s)) N_{p}(s) \mathrm{d} s \boldsymbol{e}_{i}^{*} \boldsymbol{e}_{p}^{T}, \\
& \boldsymbol{g}=\sum_{p=1}^{K} \int_{\Gamma_{\mathrm{D}}} N_{p}(s) \bar{u}(s) \mathrm{d} s \boldsymbol{e}_{p} .
\end{aligned}
$$

Equations (11) and (12) can be written in the form,

$$
\left[\begin{array}{cc}
A & C \\
D^{T} & O
\end{array}\right]\left[\begin{array}{c}
\hat{\boldsymbol{u}} \\
\hat{\boldsymbol{v}}
\end{array}\right]=\left[\begin{array}{l}
\boldsymbol{f} \\
\boldsymbol{g}
\end{array}\right] .
$$

It is (13) that is the discretized form of the Poisson problem. Especially for the case with $\psi_{i}(\boldsymbol{x})=w_{i}(\boldsymbol{x})(i=$ $1,2, \cdots, N)$, the coefficient matrix in (13) becomes asymmetric. For this case, the resulting meshless method is called the Element-Free Petrov-Galerkin (EFPG) method. On the other hand, for the case with $\psi_{i}(\boldsymbol{x})=\phi_{i}(\boldsymbol{x})(i=$ $1,2, \cdots, N)$, the coefficient matrix becomes symmetric and (13) coincides with the matrix equation for the EFG method [2]. In this sense, the above method is a complete extension of the EFG method.

\subsection{Selection of functions $N_{p}$ 's}

As mentioned above, linearly independent functions $N_{p}$ 's are required for the discretization of the essential boundary condition. As the functions, Lagrange interpolants [2] and boundary-element shape functions [6] have been so far employed. However, even if these functions are adopted for $N_{p}$ 's, the essential boundary conditions are not exactly satisfied on the boundary nodes. In fact, the following equations are only fulfilled:

$$
\int_{\Gamma_{\mathrm{D}}} N_{p}(s)(u-\bar{u}) \mathrm{d} s=0 \quad(p=1,2, \cdots, K) .
$$

In order to exactly enforce the essential boundary conditions on the boundary nodes, we propose that $\delta$-functions of an arclength $s$ be used for $N_{p}$ 's. For this case, the explicit forms of $N_{p}(s)$ 's can be written as

$$
N_{p}(s)=\delta\left(s-s_{p}\right) \quad(p=1,2, \cdots, K),
$$

where $s_{p}$ denotes an arclength to the $p$ th boundary node. Note that the substitution of (15) into (14) yields $u\left(\boldsymbol{x}\left(s_{p}\right)\right)=\bar{u}\left(s_{p}\right)$. Hence, the essential boundary conditions are exactly satisfied on the boundary nodes. In addition, the vector $g$ and the matrices, $C$ and $D$, are given by

$$
\begin{aligned}
& \boldsymbol{g}=\sum_{p=1}^{K} \bar{u}\left(s_{p}\right) \boldsymbol{e}_{p}, \\
& \boldsymbol{C}=\sum_{i=1}^{N} \sum_{p=1}^{K} \psi_{i}\left(\boldsymbol{x}\left(s_{p}\right)\right) \boldsymbol{e}_{i}^{*} \boldsymbol{e}_{p}^{T} \\
& D=\sum_{i=1}^{N} \sum_{p=1}^{K} \phi_{i}\left(\boldsymbol{x}\left(s_{p}\right)\right) \boldsymbol{e}_{i}^{*} \boldsymbol{e}_{p}^{T} .
\end{aligned}
$$

Thus, if (15) is used for the discretization of the essential boundary condition, $g, C$ and $D$ can be easily evaluated without using any numerical integrations. For example, the $(i, p)$ th element of $C$ and the $p$ th component of $\boldsymbol{g}$ are calculated as $C_{i p}=\psi_{i}\left(\boldsymbol{x}\left(s_{p}\right)\right)$ and $g_{p}=\bar{u}\left(s_{p}\right)$, respectively.

In the following, the EFG and the EFPG with (15) as $N_{p}$ 's are referred to as the collocation EFG and the collocation EFPG, respectively. In contrast, the EFG and the EFPG with conventional $N_{p}(s)$ 's are referred to as the standard EFG and the standard EFPG, respectively.

\section{Performance Evaluation}

A numerical code for solving the 2D Poisson problem has been developed on the basis of four types of meshless methods: the standard EFG, the standard EFPG, the collocation EFG and the collocation EFPG. In the present section, the accuracy of these methods is investigated by use of the code. As the measure of the accuracy, we use the relative error defined by $\epsilon \equiv\left\|u_{\mathrm{N}}-u_{\mathrm{A}}\right\| /\left\|u_{\mathrm{A}}\right\|$, where $u_{\mathrm{N}}$ and $u_{\mathrm{A}}$ are the numerical and the analytic solutions, respectively, and the maximum norm is adopted for the definition of \|\| .

Throughout the present section, the domain $\Omega$ is assumed as $\Omega=(0,1) \times(0,1)$ and the given functions, $\bar{u}, \bar{q}$ and $p$, are determined so that the analytic solution of the 2D Poisson problem may be $u=\exp \left[-\left(x^{2}+y^{2}\right)\right]$. Also, only the Dirichlet problem is solved throughout the present section.

The nodes are uniformly placed in $\Omega$. Moreover, in the MLS approximation, a linear basis $\boldsymbol{p}^{T}(\boldsymbol{x})=\left[\begin{array}{lll}1 & x & y\end{array}\right]$ and the exponential-type weight function are assumed. The explicit form of the weight function is given by

$$
\begin{aligned}
& w_{i}(\boldsymbol{x})=\omega\left(\left|\boldsymbol{x}-\boldsymbol{x}_{i}\right|\right), \\
& \omega(r) \equiv \begin{cases}\frac{\exp \left[-(r / c)^{2}\right]-\exp \left[-(R / c)^{2}\right]}{1-\exp \left[-(R / c)^{2}\right]} & (r \leq R) \\
0 & (r>R),\end{cases}
\end{aligned}
$$

where $R$ denotes a support radius and $c$ is a constant. In the present study, the value of $c$ is assumed to be equal to the minimum distance $h$ between two nodes.

By using the above weight functions $w_{i}$ 's, the shape functions $\phi_{i}$ 's [1-3] can be determined by

$$
\phi_{i}(\boldsymbol{x})=\boldsymbol{p}^{T}(\boldsymbol{x}) M^{-1}(\boldsymbol{x}) \boldsymbol{b}_{i}(\boldsymbol{x}) .
$$

Here, the matrix $M(\boldsymbol{x})$ and the vector $\boldsymbol{b}_{i}(\boldsymbol{x})$ are given by

$$
\begin{aligned}
& M(\boldsymbol{x})=\sum_{i=1}^{N} w_{i}(\boldsymbol{x}) \boldsymbol{p}\left(\boldsymbol{x}_{i}\right) \boldsymbol{p}^{T}\left(\boldsymbol{x}_{i}\right) \\
& \boldsymbol{b}_{i}(\boldsymbol{x})=w_{i}(\boldsymbol{x}) \boldsymbol{p}\left(\boldsymbol{x}_{i}\right)
\end{aligned}
$$

Note that, for the EFPG method, the weight functions $w_{i}$ 's are chosen as $\psi_{i}$ 's. On the other hand, the shape functions $\phi_{i}$ 's are adopted as $\psi_{i}$ 's for the EFG method.

Let us first investigate the influence of the support radius on the accuracy of the meshless methods. To this end, the dependence of the relative error on the support radius is numerically determined for the standard EFG/EFPG and 


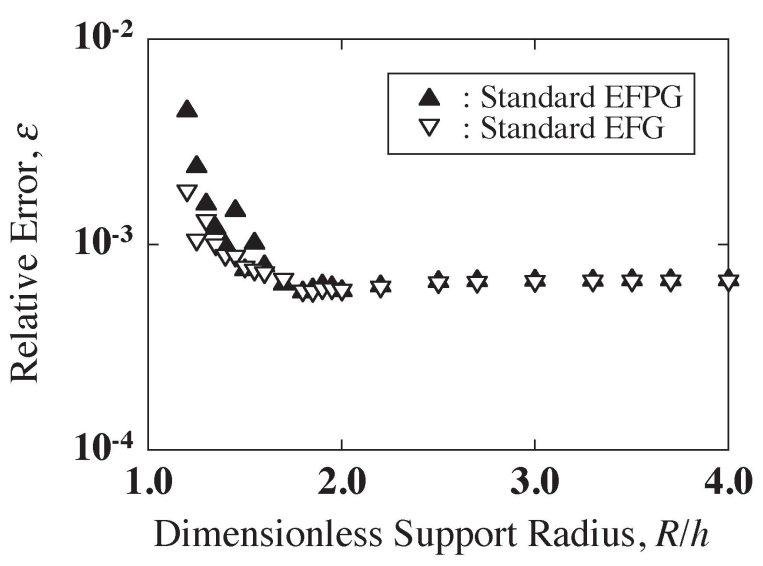

(a)

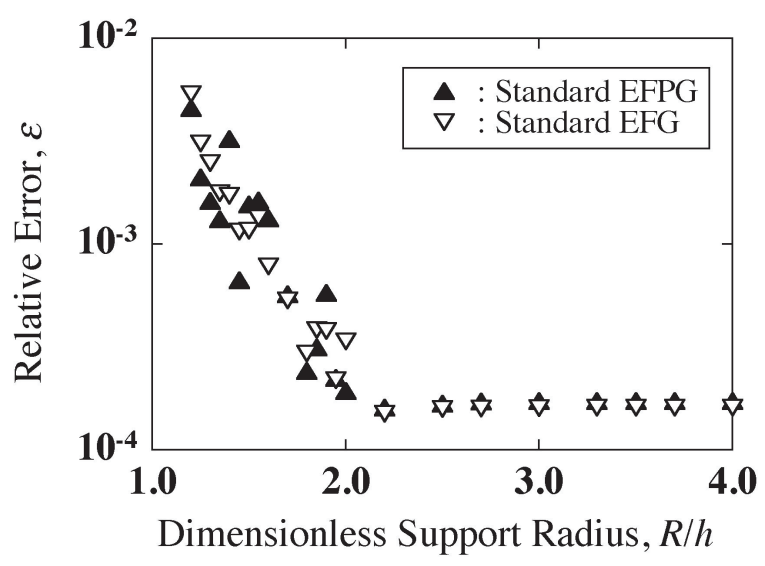

(b)

Fig. 1 Dependence of the relative error $\epsilon$ on the support radius $R$. Here, (a) $N=441$ and (b) $N=1681$.

the results of computations are depicted in Figs. 1 (a) and 1 (b). We see from these figures that the relative error decreases with $R / h$ until it becomes almost constant above a certain limit of $R / h$. In addition, the limit does not depend on the type of meshless methods but on the number of nodes. Since only the problems with $N<3000$ are solved in the present study, the value of $R / h$ is fixed as $R / h=2.5$, hereafter.

Next, we investigate how the accuracy of the meshless methods is affected by the functions $N_{p}$ 's. For this purpose, the relative error of the standard/collocation EFG is calculated as functions of the number of nodes and is depicted in Fig. 2. The convergence rate of the collocation EFG is equal to that of the standard EFG for $N<1700$, whereas it slightly decreases with $N$ for $N>1700$. The similar tendencies are also observed for the standard/collocation EFPG (see Fig. 3). The convergence rate of the standard EFPG takes a constant value. On the other hand, the convergence rate of the collocation EFPG is constant for $N<1700$ and it slightly diminishes for $N>1700$. Furthermore, Figs. 2 and 3 also show that the accuracy of the collocation meshless method is superior to that of the standard meshless method. From these results, we can con-

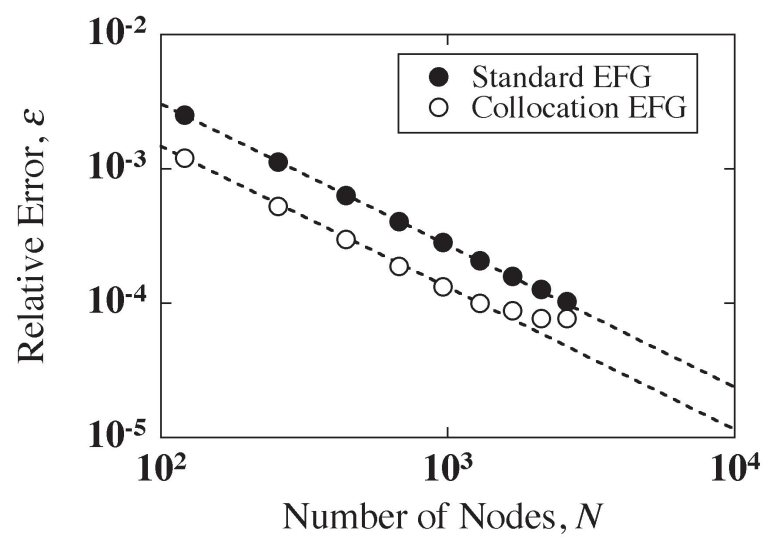

Fig. 2 The relative error $\epsilon$ as functions of the number $N$ of nodes. Here, the Poisson problem is solved by means of either the standard EFG or the collocation EFG.

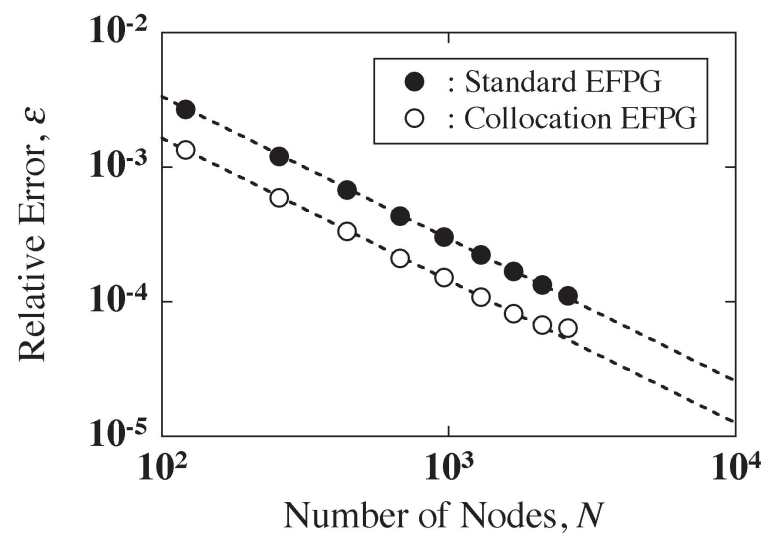

Fig. 3 The relative error $\epsilon$ as functions of the number $N$ of nodes. Here, the Poisson problem is solved by means of either the standard EFPG or the collocation EFPG.

clude that the collocation method is considerably effective for incorporating the essential boundary conditions into the EFG and the EFPG.

\section{Conclusion}

Without using the Lagrange multiplier method or the penalty method, we have formulated the meshless methods successfully. As the method for imposing the essential boundary conditions, not only the standard method but the collocation method is employed. As a result, four types of the meshless methods are derived: the standard EFG, the collocation EFG, the standard EFPG and the collocation EFPG. On the basis of these four methods, a numerical code has been developed for solving the 2D Poisson problem. By use of the code, the accuracy of these meshless methods is investigated numerically. Conclusions obtained in the present study are summarized as follows.

1) The accuracy of four kinds of meshless methods is considerably improved with an increase in the support radius. 
2) From the standpoint of the accuracy, the collocation meshless method is superior to the standard one.

\section{Acknowledgments}

This work was supported in part by Japan Society for the Promotion of Science under a Grant-in-Aid for Scientific Research (B) No. 22360042. A part of this work was also performed with the support and under the auspices of the NIFS Collaboration Research program (NIFS09KDBN003, NIFS10KNXN178).
[1] B. Nayroles, G. Touzot and P. Villon, Comput. Mech. 10, 307 (1992).

[2] T. Belytschko, Y.Y. Lu and L. Gu, Int. J. Numer. Meth. Engng. 37, 229 (1994).

[3] S.N. Atluri and T. Zhu, Comput. Mech. 22, 117 (1998).

[4] Y.X. Mukherjee and S. Mukherjee, Int. J. Numer. Meth. Engng. 40, 797 (1997).

[5] M.K. Chati, G.H. Paulino and S. Mukherjee, Int. J. Numer. Meth. Engng. 50, 2233 (2001).

[6] S. Ikuno, K. Takakura and A. Kamitani, IEEE Trans. Magn. 43, 1501 (2007). 